\title{
Häufiger als bisher angenommen?
}

Fragestellung: Die retrospektive Analyse untersuchte, wie häufig die behandlungsinduzierte Neuropathie bei Diabetes mellitus („Treatment-induced Neuropathy in Diabetes“, TIND) vorkommt und welche Risikofaktoren es dafür gibt.

Hintergrund: Die behandlungsinduzierte Neuropathie bei Diabetes mellitus ist schon seit langem bekannt und wurde früher auch „Insulin-Neuritis“ genannt [1]. Es handelt sich um eine schmerzhafte und autonome Neuropathie, die dann auftritt, wenn bei Patienten mit langjährigem Diabetes mellitus und Polyneuropathie das glykosylierte Hämoglobin $\left(\mathrm{HbA}_{1 c}\right)$ rasch gesenkt wird. Die Patienten leiden unter brennenden und einschießenden Schmerzen sowie zum Teil an einer orthostatischen Hypotonie mit Synkopen.

Patienten und Methodik: Im Rahmen der retrospektiven Studie wurden die Daten von 910 Patienten ausgewertet. Die folgenden Kriterien wurden verwendet, um eine Diagnose der behandlungsinduzierten Neuropathie zu stellen: Abnahme des $\mathrm{HbA}_{\mathrm{lc}}$ um mehr als zwei Prozentpunkte in drei Monaten, akuter Beginn neuropathischer Schmerzen (> 3 Punkte Erhöhung auf einer 11-Punkte-Likert-Skala) und/oder Entwicklung einer autonomen Dysfunktion über zwei Wochen, die schwerwiegend genug war, einen Arztbesuch

Gibbons CH, Freeman R. Treatment-induced neuropathy of diabetes: an acute, iatrogenic complication of diabetes. Brain 2015; $138: 43-52$ zu initiieren, sowie Beginn der neuropathischen Schmerzen und/oder autonomen Symptome innerhalb von acht Wochen nach der Abnahme des $\mathrm{HbA}_{1 \mathrm{c}}$.
Ergebnisse: Von 168 Patienten, deren $\mathrm{HbA}_{\mathrm{lc}}$ innerhalb von drei Monaten um mehr als zwei Prozentpunkte abgesenkt wurde, erlitten 104 (10,9\%) eine behandlungsinduzierte Neuropathie. Unter den Patienten mit langsamerem Absinken des $\mathrm{HbA}_{\mathrm{lc}}$ waren dies nur 32 (4,3\%). Wurde der $\mathrm{HbA}_{1 \mathrm{c}}$ innerhalb von drei Monaten um vier Prozentpunkte gesenkt, erlitten sogar $80 \%$ der $\mathrm{Pa}$ tienten diese Komplikation. Gewichtsverlust schien ein zusätzlicher Risikofaktor zu sein. Mit welchem Medikament die Blutzuckerregulierung erfolgt war, spielte keine Rolle. Fast alle Patienten litten unter neuropathischen Schmerzen, die sie zuvor nur in geringem Ausmaß oder gar nicht hatten. Die klinische Neurophysiologie ergab meist keine oder nur milde Auffälligkeiten. Die Verschlechterung der autonomen Funktion konnte hingegen bei einer Subgruppe auch apparativ nachgewiesen werden.

Schlussfolgerungen: Die Autoren schließen daraus, dass die behandlungsinduzierte Neuropathie bei Diabetes häufiger als angenommen und abhängig von der Geschwindigkeit der $\mathrm{HbA}_{1 c^{-}}$ Korrektur ist. Ursächlich werden ein endoneurales Ödem, das sich durch die Öffnung epineuraler arterio-venöser Shunts ausbildet, Apoptose durch Glukosedeprivation oder mikrovaskuläre neuronale Schäden diskutiert. Der zusätzliche Risikofaktor des Gewichtsverlusts weist auf einen möglichen Vitaminmangel oder auf eine Vermehrung proinflammatorischer Zytokine hin. Es wird eine symptomatische Therapie empfohlen und es heißt, dass sich die Beschwerden mit der Zeit bessern. Die Autoren bemerken einschränkend, dass die Häufigkeit der behandlungsinduzierten Neuropathie in dieser Studie überschätzt worden sein könnte, da der Patienteneinschluss in einem tertiären und nicht in einem primären Zentrum für Diabetesversorgung erfolgte.

\section{- Kommentar von Claudia Sommer, Würzburg}

\section{Blutzucker besser langsam normalisieren?}

Die Studie macht einen wichtigen Punkt, indem sie die Gefahren der allzu schnellen Normalisierung der Stoffwechsellage bei Diabetikern aufweist. Über $10 \%$ der Patienten erlitten hierbei eine schmerzhafte autonome Neuropathie, was früher auch als "Insulin-Neuritis" bezeichnet wurde [2]. Noch ist unklar, über welchen Zeitraum und zu welchem Grad sich diese Neuropathie zurückbildet. Die Prognose gilt generell als gut und die Erkrankung gilt als monophasisch, aber das Ausmaß der möglichen Folgeschäden ist nicht genau bekannt [3], zumal sich auch die Retinopathie unter der raschen Blutzuckersenkung verschlimmern kann [4]. Ob die Zahlen wirklich so hoch sind, bleibt unklar, da es sich hier um eine ausgewählte Population von Patienten mit Diabetes und Neuropathie oder Verdacht auf Neuropathie handelte, die einem hochspezialisierten Zentrum zugewiesen wurde. Dennoch, selbst wenn das Risiko in der Allgemeinheit von Menschen mit Diabetes nur halb so groß oder ein Drittel so groß wäre, lohnt die Achtsamkeit in der Behandlung.
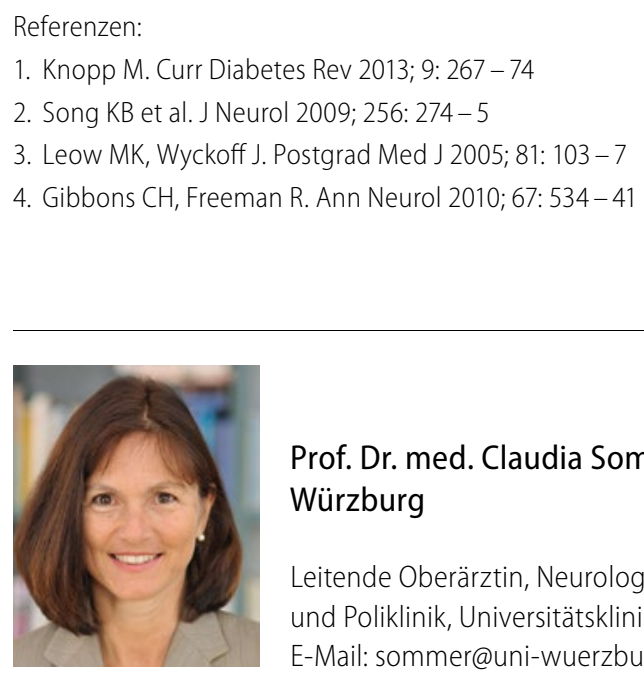

Prof. Dr. med. Claudia Sommer, Würzburg

Leitende Oberärztin, Neurologische Klinik und Poliklinik, Universitätsklinikum Würzburg E-Mail:sommer@uni-wuerzburg.de 